\title{
Methods for studying close-track efficiency
}

\author{
M.D. Mestayer ${ }^{\mathrm{a}}$, K.R Mikhailov ${ }^{\mathrm{b}}$, A.V. Stavinskiy ${ }^{\mathrm{b}}$, A.V. Vlassov ${ }^{\mathrm{b}, *}$ \\ ${ }^{a}$ Thomas Jefferson National Accelerator Facility, 12000 Jefferson Avenue, Newport News VA 23606, USA \\ ${ }^{\mathrm{b}}$ ITEP, B. Cheremushkinskaya 25, Moscow 117218, Russia
}

Received 16 June 2003; received in revised form 26 January 2004; accepted 29 January 2004

\begin{abstract}
Wire chambers used for particle tracking suffer a loss of efficiency when the trajectories of two particles from the same event are very close together in space. We describe two new methods for the study of this close-track efficiency. One is based on the study of a correlation function for particles with different masses as a function of their relative momenta in the laboratory reference system. The other method is based on the analysis of artificial events, constructed by merging raw data from separate events. Both methods and the standard Monte Carlo method were applied to data from the CLAS detector at Jefferson Laboratory. All three methods provide the same result for close-track efficiency with an accuracy sufficient for practical application.
\end{abstract}

(C) 2004 Elsevier B.V. All rights reserved.

PACS: 25.75.Gz; 29.40.Gx; 29.85.+c

Keywords: Close-track efficiency; Track reconstruction; Particle correlations

\section{Introduction}

Some important physics tasks need both particle identification and precise momentum measurement for an extremely difficult configuration: two particles with the same charge and similar momenta. It is well-known that the correlations of particles with small relative velocities (which means close particle momenta for identical particles) are sensitive to the space-time intervals between the emission points, due to quantum interference effects and to the strong and Coulomb

\footnotetext{
*Corresponding author. Thomas Jefferson National Accelerator facility, 12000 Jefferson Avenue, Newport News VA 23606, USA.

E-mail address: vlassov@jlab.org (A.V. Vlassov).
}

final state interactions [1]. Finite two-track resolution restricts two-particle correlation measurements at small relative momenta, because both particles can hit the same or neighboring detector cells. As a rule, the probability to lose at least one of the two tracks is higher if those tracks are close to each other.

The traditional method for studying close-track efficiency is based on Monte Carlo simulations, most frequently in the GEANT [2] framework. Any simulation is an approximation of the real detector properties, and the better the detector description, the more CPU time is needed to produce (simulate) one event. In the proposed new methods we tried to use the data from real events from the detector to make the efficiency estimate faster and more reliable. 
One of the new methods, the different mass method, is based on the hypothesis that the narrow singularities from secondary particle interactions are expected in the region of small particle momentum difference in the center-of-mass reference frame (CMRF), while loss of pairs due to track inefficiency is expected in the region of small particle momentum difference in the laboratory reference frame (LRF). In the case of particles with different masses, these two regions are separated. This means that one can interpret a sharp decrease of a correlation function for particles with different masses at small relative momenta in the laboratory reference system as a close track efficiency effect [3].

The other new method, the event merging method, uses artificial events constructed from two real events, using the original information from the detectors (raw data information). After selecting two events, with well-identified protons whose momenta are close to each other, the information from both events is merged into another (artificial) event, resulting in an event with a pair of protons with small relative momentum. These new events were reconstructed using the same standard procedure. The probability of pair reconstruction is a measure of the close-track efficiency, because both tracks had been reconstructed separately. Both methods are described in detail in a technical note [4].

\section{Experimental setup and definitions}

New methods were developed for the study of the close-track efficiency for the CLAS [5] detector in the Thomas Jefferson National Accelerator Facility (Jlab). These methods are general enough that they can be applied to other detectors.

The CLAS detector in Hall $\mathrm{B}$ is a six-sector toroidal magnetic spectrometer. The magnetic field is generated by six iron-free superconducting coils. The detection systems consist of three drift chamber (DC) regions per sector to determine the trajectories of charged particles [6,7], scintillator counters (TOF) for the trigger and time-offlight measurements [8], Cherenkov counters to distinguish between electrons and negative pions
[9], and electromagnetic shower calorimeters (EC) to identify electrons, photons and neutrons $[10,11]$.

To study the close track efficiency we have used the data from the " $E 2$ " run at energy $4.46 \mathrm{GeV}$ $\left(\mathrm{e}+\mathrm{A} \rightarrow \mathrm{e}^{\prime} \mathrm{h}_{1} \mathrm{~h}_{2}+\mathrm{X}\right.$, where A are ${ }^{3} \mathrm{He},{ }^{4} \mathrm{He},{ }^{12} \mathrm{C}$, ${ }^{56} \mathrm{Fe}$, and $\mathrm{h}_{i}$ are $\left.\pi, \mathrm{p}, \mathrm{d}\right)$. The electron beam current was typically about $10 \mathrm{nA}$, which yielded a nominal luminosity of about $10^{34} \mathrm{~cm}^{-2} \mathrm{~s}^{-2}$. The magnetic field was $50 \%$ of the maximum value.

We define the two-hadron correlation function $R$ for fixed $Q^{2}, v$ of the scattered electron as

$R(\vec{q}, \vec{p})=\frac{\mathrm{d}^{2} \sigma}{\mathrm{d} \vec{p}_{1} \cdot \mathrm{d} \vec{p}_{2}} /\left(\frac{\mathrm{d} \sigma}{\mathrm{d} \vec{p}_{1}} \cdot \frac{\mathrm{d} \sigma}{\mathrm{d} \vec{p}_{2}}\right)$

where $\vec{p}_{i}$ is the individual hadron momentum, and $\vec{q}=\vec{p}_{1}-\vec{p}_{2}$ and $\vec{p}=\left(\vec{p}_{1}+\vec{p}_{2}\right) / 2$ are the pair momentum difference and mean momentum, respectively.

The measured cross-section is affected by the detector efficiency:

$\frac{\mathrm{d} \sigma_{\text {measured }}}{\mathrm{d} \vec{p}_{i}}=\varepsilon_{1}\left(\vec{p}_{i}\right) \cdot \frac{\mathrm{d} \sigma}{\mathrm{d} \vec{p}_{i}}$

$\frac{\mathrm{d}^{2} \sigma_{\text {measured }}}{\mathrm{d} \vec{p}_{1} \cdot \mathrm{d} \vec{p}_{2}}=\varepsilon_{2}\left(\vec{p}_{1}, \vec{p}_{2}\right) \cdot \frac{\mathrm{d}^{2} \sigma}{\mathrm{d} \vec{p}_{1} \cdot \mathrm{d} \vec{p}_{2}}$

We are interested in the unfactorized part of the pair efficiency $\varepsilon(\vec{p}, \vec{q})$, which is considered to be the close-track efficiency:

$\varepsilon(\vec{q}, \vec{p})=\frac{\varepsilon_{2}\left(\vec{p}_{1}, \vec{p}_{2}\right)}{\varepsilon_{1}\left(\vec{p}_{1}\right) \cdot \varepsilon_{1}\left(\vec{p}_{2}\right)}$.

As seen from Eqs. (1)-(4) the close-track efficiency $\varepsilon(\vec{q}, \vec{p})$ can be defined as

$\varepsilon(\vec{q}, \vec{p})=\frac{R_{\text {measured }}(\vec{q}, \vec{p})}{R_{\text {ideal }}(\vec{q}, \vec{p})}$

where $R_{\text {ideal }}(\vec{q}, \vec{p})$ is the two-hadron correlation function as seen with an ideal detector. For the rest of this paper, we consider the efficiency $\varepsilon$ and correlation function $R$ to be functions of the norms of $\vec{q}$ and $\vec{p}$ only: $\varepsilon \equiv \varepsilon(q, p)$, and $R \equiv R(q, p)$.

For a fixed magnetic field, the track proximity depends not only on $q$, but also on $p$. This is a reason to expect some $p$ dependence of the closetrack efficiency. 


\section{The study of close-track efficiency based on the experimental data on $\pi^{+} p$ and pd correlations (the different mass method)}

The experimental difficulty in the detection of identical particles with small relative momenta $\vec{q}$ is associated with the fact that the gaps between their tracks are small not only near the interaction point, but also throughout their length. If the particles have the same charge and close momenta in the laboratory reference system, but differ significantly in mass, their track proximity will be the same as for identical particles and so the closetrack efficiency is expected to be the same. Here we neglect a change in momenta due to the ionization loss and other effects dependent on the particle velocities.

For a pair of particles with different masses, the Coulomb and strong final-state interaction can also be important, but only in the kinematical region characterized by small relative velocities, which means small relative momenta in the centerof-mass reference frame (CMRF) and invariant masses near threshold [12]. To the best of our knowledge, there is no reason for sharp singularities to appear in the correlation function of particles with different masses at small relative momenta $\vec{q}$ in the laboratory reference frame (LRF) and no experimental evidence for their existence is available. We assume that such singularities are negligible.

To calculate the $\mathrm{p} \pi^{+}$correlation function we used the well-known "mixing" procedure [13]

$R(q)=N_{\mathrm{r}}(q) / N_{\mathrm{m}}(q)$

where $N_{\mathrm{r}}$ is a distribution of $\mathrm{p} \pi^{+}$pairs from real events, and $N_{\mathrm{m}}$ is distribution from "mixed" events, where the proton and the pion are from different independent events.

The measured $q$ dependences of the $\mathrm{p} \pi^{+}$ correlation functions for $\mathrm{eA} \rightarrow \mathrm{e}^{\prime} \mathrm{p} \pi^{+} \mathrm{X}$ reaction at $4.46 \mathrm{GeV}$ are shown in Fig. 1. The decrease of the correlation function at small $q$ was interpreted as the two-track efficiency's dependence on $q$. One also can see the smooth growth of the correlation function at larger $q$.

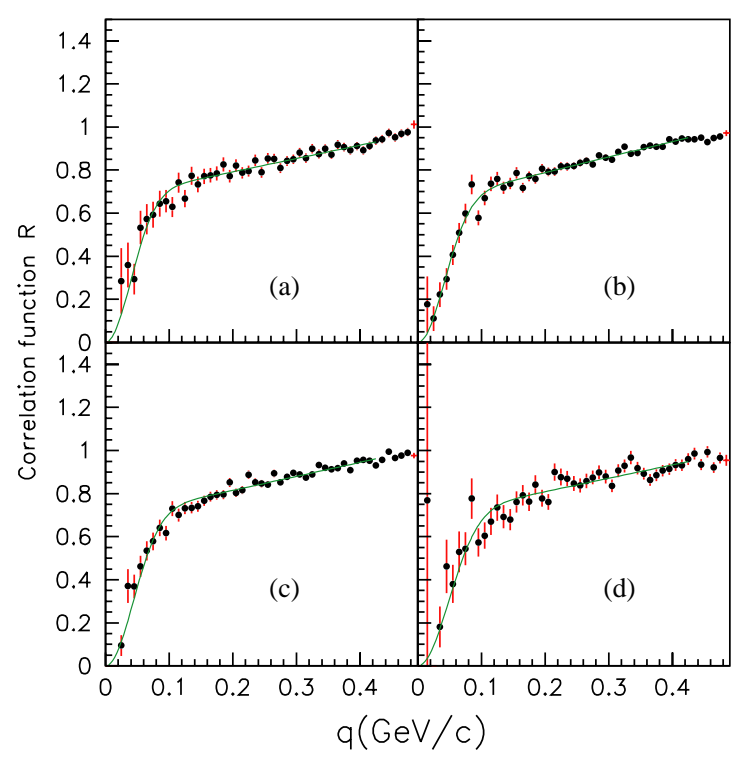

Fig. 1. The $\mathrm{p} \pi^{+}$correlation function versus the relative momenta q. a, b, c, d stand for the ${ }^{3} \mathrm{He},{ }^{4} \mathrm{He},{ }^{12} \mathrm{C}$ and ${ }^{56} \mathrm{Fe}$ targets, respectively. The curves represent the best fit (Eq. (7)) within the range $q<0.43 \mathrm{GeV} / c$. The mean momentum of the particles is $0.42 \mathrm{GeV} / c$.

We fitted the efficiency with the function:

$\varepsilon(q)=a \cdot(1+b q) \cdot\left(1-\exp \left(-\frac{q^{2}}{\varepsilon_{0}^{2}}\right)\right)$.

Here $a$ is a normalization constant, $b$ corresponds to the smooth efficiency dependence at the relatively large momenta and $\varepsilon_{0}$ is the width of the Gaussian corresponding to the inefficiency at small relative momenta. Several factors (violation of the energy and momentum conservation in the mixed pairs, a possible smooth dependence of the detector efficiency on $q$, etc.) lead to a slow growth in the correlation functions with $q$.

The results of the data fitting using Eq. (7) are presented in Table 1. One can see that the parameter $\varepsilon_{0}$ does not depend on the mass number of the target A within the statistical errors, and increases with the particle momenta $p$. The same is true for the pd correlation function (see Fig. 2 and Table 1). For similar $p(0.48$ and $0.44 \mathrm{GeV} / c)$ the parameters $\varepsilon_{0}$ for $\mathrm{p} \pi^{+}$and $\mathrm{pd}$ are the same within the errors.

To estimate possible systematic errors in the data, we vary the upper value of $q_{\max }$ of the fitting 
Table 1

Results of fits for $\mathrm{p} \pi^{+}$and pd correlation functions dependencies on $q$

\begin{tabular}{llllll}
\hline$\langle p\rangle(\mathrm{GeV} / c)$ & $\mathrm{pair}$ & $\mathrm{A}$ & Fit & $q_{\max }(\mathrm{GeV} / c)$ & $\varepsilon_{0}(\mathrm{Gev} / c)$ \\
\hline 0.42 & $\mathrm{p} \pi^{+}$ & $\mathrm{C}$ & $\mathrm{I}$ & 0.31 & $0.054 \pm 0.003$ \\
0.42 & $\mathrm{p} \pi^{+}$ & $\mathrm{C}$ & $\mathrm{I}$ & 0.35 & $0.056 \pm 0.003$ \\
0.42 & $\mathrm{p} \pi^{+}$ & $\mathrm{C}$ & $\mathrm{I}$ & 0.39 & $0.058 \pm 0.003$ \\
0.42 & $\mathrm{p} \pi^{+}$ & $\mathrm{C}$ & $\mathrm{I}$ & 0.43 & $0.059 \pm 0.003$ \\
0.42 & $\mathrm{p} \pi^{+}$ & $\mathrm{C}$ & $\mathrm{I}$ & 0.47 & $0.060 \pm 0.003$ \\
& & & & & \\
0.34 & $\mathrm{p} \pi^{+}$ & $\mathrm{He}^{3}$ & $\mathrm{I}$ & 0.43 & $0.042 \pm 0.007$ \\
0.34 & $\mathrm{p} \pi^{+}$ & $\mathrm{He}$ & $\mathrm{I}$ & 0.43 & $0.053 \pm 0.004$ \\
0.34 & $\mathrm{p} \pi^{+}$ & $\mathrm{C}$ & $\mathrm{I}$ & 0.43 & $0.052 \pm 0.004$ \\
0.34 & $\mathrm{p} \pi^{+}$ & $\mathrm{Fe}$ & $\mathrm{I}$ & 0.43 & $0.064 \pm 0.008$ \\
& & & & & \\
0.48 & $\mathrm{p} \pi^{+}$ & $\mathrm{He}$ & $\mathrm{I}$ & 0.43 & $0.066 \pm 0.005$ \\
0.48 & $\mathrm{p} \pi^{+}$ & $\mathrm{He}$ & $\mathrm{I}$ & 0.43 & $0.065 \pm 0.004$ \\
0.48 & $\mathrm{p} \pi^{+}$ & $\mathrm{C}$ & $\mathrm{I}$ & 0.43 & $0.068 \pm 0.004$ \\
0.48 & $\mathrm{p} \pi^{+}$ & $\mathrm{Fe}$ & $\mathrm{I}$ & 0.43 & $0.071 \pm 0.011$ \\
& & & & & \\
0.44 & $\mathrm{pd}$ & $\mathrm{C}$ & $\mathrm{I}$ & 0.43 & $0.074 \pm 0.010$ \\
0.44 & $\mathrm{pd}$ & $\mathrm{Fe}$ & $\mathrm{I}$ & 0.43 & $0.068 \pm 0.011$ \\
0.60 & $\mathrm{pd}$ & $\mathrm{C}$ & $\mathrm{I}$ & 0.43 & $0.079 \pm 0.011$ \\
0.60 & $\mathrm{pd}$ & $\mathrm{Fe}$ & $\mathrm{I}$ & 0.43 & $0.078 \pm 0.014$ \\
& & & & & \\
0.60 & $\mathrm{p} \pi^{+}$ & $\mathrm{C}$ & $\mathrm{II}$ & 0.43 & $0.053 \pm 0.003$ \\
0.60 & $\mathrm{p} \pi^{+}$ & $\mathrm{C}$ & $\mathrm{I}$ & 0.43 & $0.059 \pm 0.003$ \\
0.60 & $\mathrm{p} \pi^{+}$ & $\mathrm{C}$ & $\mathrm{III}$ & 0.43 & $0.067 \pm 0.003$ \\
0.60 & $\mathrm{p} \pi^{+}$ & $\mathrm{He}$ & $\mathrm{II}$ & 0.43 & $0.052 \pm 0.003$ \\
0.60 & $\mathrm{p} \pi^{+}$ & $\mathrm{He}$ & $\mathrm{I}$ & 0.43 & $0.057 \pm 0.003$ \\
0.60 & $\mathrm{p} \pi^{+}$ & $\mathrm{He}$ & $\mathrm{III}$ & 0.43 & $0.063 \pm 0.003$ \\
\hline$p 0$ & & & & &
\end{tabular}

$p$ is an average particle momentum at small $q(q<0.1 \mathrm{GeV} / c)$. $q_{\max }$ is an upper limit of the fitting interval. The last column shows the fitted value of $\varepsilon_{0}$ and its statistical error.

interval $0<q<q_{\max }$ and use two other fitting functions with different behavior at large $q$ :

$\varepsilon(q)=a \cdot(1+b \sqrt{q}) \cdot\left(1-\exp \left(-\frac{q^{2}}{\varepsilon_{0}^{2}}\right)\right)$

and

$\varepsilon(q)=a \cdot\left(1+b q^{2}\right) \cdot\left(1-\exp \left(-\frac{q^{2}}{\varepsilon_{0}^{2}}\right)\right)$.

We will refer to fits using Eqs. (7), (8) and (9) as fit I, fit II and fit III, respectively.

The sample results for different $q_{\max }$ are presented at the top of Table 1 for carbon data. One can see that the parameters are stable within the errors for $q_{\max }>0.4 \mathrm{GeV} / c$. The bottom rows of Table 1 show the results for different fitting

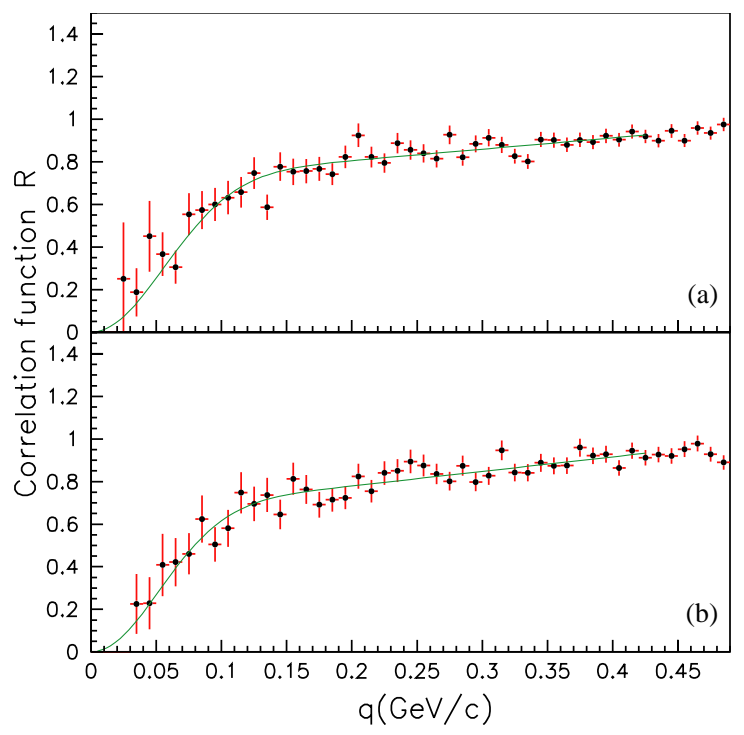

Fig. 2. Correlation function for pd as a function of relative momenta $q$. a- $-{ }^{12} \mathrm{C} ; \mathrm{b}-{ }^{56} \mathrm{Fe}$ targets, respectively. The mean momentum of a particle is $0.51 \mathrm{GeV} / c$.

functions, - fits (7)-(9), for $\mathrm{C}$ and $\mathrm{He}^{4}$ data. One can see that the parameter $\varepsilon_{0}$ obtained with the different fitting methods is constant within about $10 \%$. We consider this difference to be an estimate of our systematic uncertainties.

We used fit $\mathbf{I}$ and $q_{\max }=0.43$ for the $\varepsilon_{0}$ estimate. Averaged for different $\mathrm{A}$, our final results for $\varepsilon_{0}$ are:

for $\mathrm{p} \pi^{+},\langle p\rangle=0.34 \mathrm{GeV} / c, \varepsilon_{0}=0.052 \pm 0.003$ $\pm 0.005 \mathrm{GeV} / c$,

for $\mathrm{p} \pi^{+},\langle p\rangle=0.48 \mathrm{GeV} / c, \varepsilon_{0}=0.067 \pm 0.002$ $\pm 0.007 \mathrm{GeV} / c$

for $\mathrm{pd},\langle p\rangle=0.44 \mathrm{GeV} / c, \varepsilon_{0}=0.071 \pm 0.007$ $\pm 0.007 \mathrm{GeV} / c$

for $\mathrm{pd},\langle p\rangle=0.60 \mathrm{GeV} / c, \varepsilon_{0}=0.079 \pm 0.009$ $\pm 0.008 \mathrm{GeV} / c$.

\section{The "event merging" method for close-track efficiency study}

The idea of this method is to use raw data from the detector for events with reconstructed single protons for producing artificial events with proton pairs. Then standard reconstruction procedures were applied to these "merged" events. Because 
the reconstruction procedure for single proton events and for proton pair events is the same, and since the single protons had been reconstructed, the inefficiency due to close tracks can be evaluated.

The exact procedure was as follows:

The initial information was the file with the reconstructed events, which contained both raw data information and reconstructed information such as the type of secondary particle, its momentum, the track parameters, the hit TOF scintillator number, the reconstructed start time of the event, etc.

The start time is defined as the time when the scattered electron left the target. It is determined by identifying the electron candidate and calculating the track length from the target to the detector and subtracting this time of flight from the electron's TOF signal. We used the data from the E2 run, and in particular, the electron-carbon interactions at $4.46 \mathrm{GeV}$.

The track reconstruction procedure is independent for each of the six sectors of the CLAS, and the $q$ for protons from different sectors is large. So to study the proton pair efficiency we selected events with a well-reconstructed proton with a good track quality in a particular sector of the CLAS, say sector i. Trigger conditions demand also a scattered electron in the event, so we select events with a reconstructed electron in some other sector $\mathbf{j} \neq \mathbf{i}$. The proton momentum was required to exceed $0.3 \mathrm{GeV} / c$.

From the data sample specific pairs of events were selected, where

(a) the momentum of the reconstructed protons are close, so that the momentum difference $q<0.4 \mathrm{GeV} / c$, which is sufficient for our efficiency study.

(b) the proton tracks from those events do not hit the same TOF scintillator. If in a real event a pair of particles hit the same scintillator, the measured TOF is wrong for both of them, and the event was discarded from the analysis.

For those event pairs ( $A$ and $B$ ) a new event (event $C$ ) was constructed, where the raw data information of sector $\mathbf{i}$ was the sum of data from event $A$ and $B$, i.e., event $B$ is merged into event $A$.
The merging of the two events included the following steps:

(a) Event $C$ was mostly the same as event $A$. Only the DC and TOF information for sector $\mathbf{i}$ from event $B$ was merged into event $A$. The other detectors do not influence the proton reconstruction procedure in this sector.

(b) The TOF values and the drift times for event $B$ were corrected by the start time difference of events $B$ and $A$.

(c) The DC information of event $B$ can involve the same wire numbers as in event $A$. In this case the drift times for those wires were modified to be an average of events $B$ and $A$. We verified that if we used the time values from event $A$ instead, the result was the same.

As a result, we have a set of events with close proton pairs, and each proton of a pair had been reconstructed separately by the same reconstruction procedure. Possible reconstruction inefficiency in the merged events is due to the small momentum difference of those tracks. The closetrack efficiency was defined according to Eq. (5). For CLAS all six sectors are of the same quality, and the estimated efficiency was the same within errors.

It should be noted that when we merge the hits from two events, then the DC occupancy is generally increased, which can result in a lower track efficiency. But usually the DC occupancy in the CLAS is of the order of $1 \%$ and it does not affect the efficiency significantly.

Fig. 3 shows an example of the estimated close track efficiency function for mean pair momenta $p=0.48 \mathrm{GeV} / c$. The plot shows the evaluated efficiency as a function of the proton momentum difference $q$. The curve, as in the previous sections, is fit using Eq. (7).

The method is good also from the statistical point of view-we can construct enough events for the statistical errors to be negligible.

To study the efficiency dependence on the mean proton momentum $p$ for $q<0.1$ we divided the momentum range into three regions. Table 2 shows the parameters $\varepsilon_{0}$ for the different average proton momenta $p$. The systematic error was 


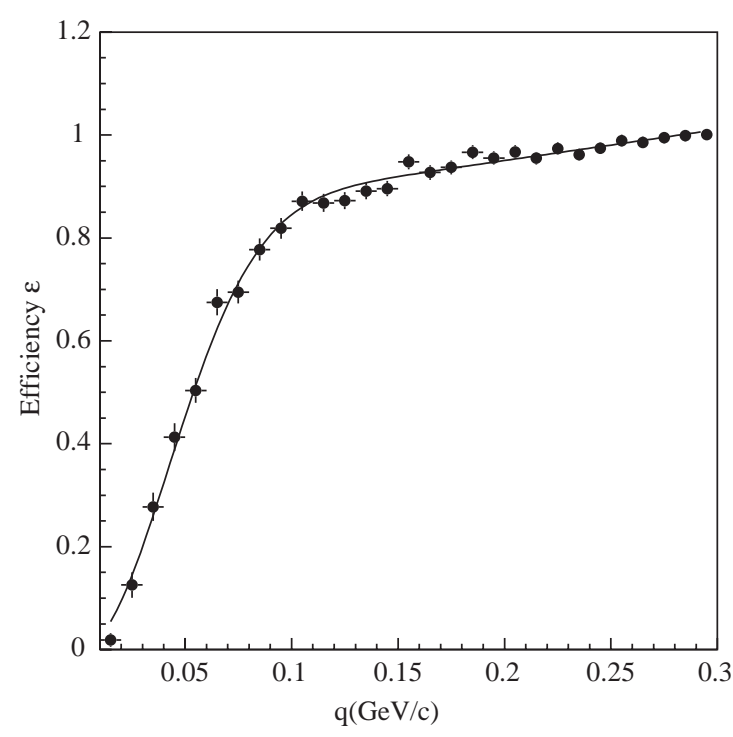

Fig. 3. The efficiency of the proton pair reconstruction as a function of their momentum difference $q$ for mean pair momenta $p=0.48 \mathrm{GeV} / c$. The curve is a fit (7).

Table 2

The efficiency parameter $\varepsilon_{0}$ for different average proton momenta

\begin{tabular}{lll}
\hline Pair & $\langle p\rangle(\mathrm{GeV} / c)$ & $\varepsilon_{0} \pm$ stat.err. \pm syst.err. \\
\hline pp & 0.396 & $0.051 \pm 0.002 \pm 0.003$ \\
pp & 0.586 & $0.066 \pm 0.003 \pm 0.004$ \\
pp & 0.774 & $0.080 \pm 0.005 \pm 0.005$ \\
\hline
\end{tabular}

estimated in analogy with the previous section by different types of fitting.

\section{Studies using simulated events}

In addition to these two methods the close-track efficiency was also estimated using Monte-Carlo simulation with the standard CLAS GEANT simulation package-GSIM [14].

Two types of secondary particle generators were used. For the first type we have simulated the reaction $e+2 \mathrm{p} \rightarrow e^{\prime}+2 \mathrm{p}+2 \pi$ using the phase space generator GENBOD [15]. To better reproduce the experimental spectra of the protons, we explored a second type of generator for GSIM simulation. In the second generator two-proton

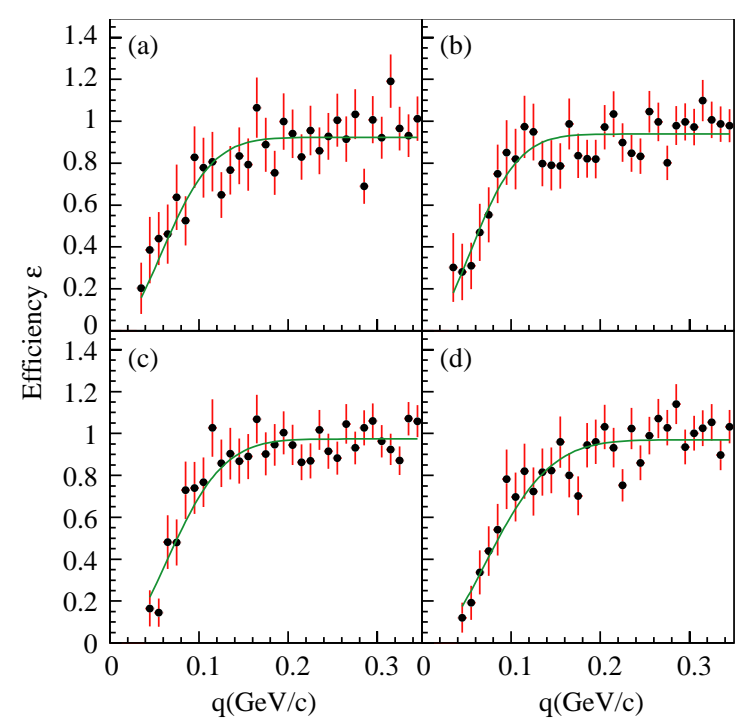

Fig. 4. The proton-proton close-track efficiency within GSIM for the different pair momentum range.

events were generated with a pair momentum spectrum similar to the experimental one. We have observed only a weak dependence on choice of event generators.

We separated the pair momentum range into four regions to study the efficiency dependence as a function of the pair momentum $p$. The mean pair momentum of the four bins, for $q<0.1 \mathrm{GeV} / c$, was, respectively: (a) $0.39 \mathrm{GeV} / c$, (b) $0.51 \mathrm{GeV} / c$, (c) $0.63 \mathrm{GeV} / c$ and (d) $0.80 \mathrm{GeV} / c$.

Fig. 4 shows the two-track efficiency plotted versus pair momentum difference $q$ for these four $p$ ranges. The fits to Eq. (7) match the data within the errors. The efficiency parameters $\varepsilon_{0}$ and $\chi^{2}$ of the fits are shown in Table 3. One can see that the efficiency parameter $\varepsilon_{0}$ is increasing with $\langle p\rangle$.

\section{Discussion: relative merits of the different methods}

In order to study the detector efficiency at small relative momenta, one needs the following conditions:

(a) use of a well-known input correlation function (real or simulated); 
Table 3

The efficiency parameter $\varepsilon_{0}$ for $\mathrm{pp}, \pi \mathrm{p}$ and $\pi \pi$ systems. The result of GSIM studying, using the GENBOD generator for $e+2 \mathrm{p} \rightarrow e^{\prime}+2 \mathrm{p}+2 \pi$ reaction

\begin{tabular}{llll}
\hline Pair & $\langle p\rangle(\mathrm{GeV} / c)$ & $\varepsilon_{0}(\mathrm{GeV} / c)$ & $\chi^{2} / n d f$ \\
\hline pp & 0.39 & $0.062 \pm 0.011 \pm 0.005$ & $24 / 32$ \\
pp & 0.51 & $0.066 \pm 0.008 \pm 0.003$ & $21 / 32$ \\
pp & 0.63 & $0.085 \pm 0.007 \pm 0.002$ & $27 / 31$ \\
pp & 0.80 & $0.090 \pm 0.011 \pm 0.006$ & $31 / 31$ \\
& & & \\
$\pi p$ & 0.40 & $0.078 \pm 0.007 \pm 0.007$ & $37 / 32$ \\
$\pi \mathrm{p}$ & 0.58 & $0.095 \pm 0.011 \pm 0.009$ & $31 / 32$ \\
$\pi \pi$ & 0.20 & $0.061 \pm 0.007 \pm 0.008$ & $39 / 25$ \\
$\pi \pi$ & 0.36 & $0.070 \pm 0.007 \pm 0.005$ & $21 / 29$ \\
\hline
\end{tabular}

(b) the hardware model should be as close to the real detector as possible, and

(c) the software should be identical to the real procedure used for event reconstruction and calculation of the correlation function.

Let us consider to what extent those conditions are fulfilled for the three methods. While all three methods provide compatible results, the nature of possible systematic errors for them is different.

Since $\mathrm{p} \pi^{+}$and $\mathrm{pd}$ pairs are not free from physical sources of correlations, it can lead in principle to some systematic uncertainties. For the two other methods, the correlation functions are known. The advantage of this method is that the real detector response for pair production is used. Most of the hardware properties are taken into account in the event merging method as well, but possible cross-talk between nearby channels is omitted in the GEANT and event merging methods.

One can simulate the detector properties using GEANT to some level of accuracy only. The event merging method does it automatically, although there is a question of how to take into account the real detector response in the case of overlapping hits.

The obvious advantage of the high statistical accuracy of event merging method allows one to evaluate the efficiency as a function of different variables, like particle momenta and emission angle. In GEANT simulations the differential close-track efficiency can also be studied, but the detailed description of the detector requires a lot of CPU time. The statistical accuracy for the different mass method is limited by the real number of events with different mass pairs, which is comparable with the statistics for identical pairs.

The procedure for event reconstruction and calculation of the correlation function was the same for all three methods.

For comparison, Fig. 5 shows the efficiency estimated by all methods for particles with mean momentum $p=0.5 \mathrm{GeV} / c$. One can see that all methods are in good agreement.

Again we found that the efficiency parameter $\varepsilon_{0}$ is increasing with average particle momenta, which seems natural, because for a fixed particle momentum difference $q$, tracks should be closer in the laboratory frame for larger momentum.

The other thing one can expect for magnetic tracking detectors is that the efficiency dependents on the magnetic field integral. For CLAS that means a possible dependence on the particle emission angle. Fig. 6 shows the angular dependence of $\varepsilon_{0}$ at $p=0.4 \mathrm{GeV} / c$. The curve in the figure is drawn by eye. A small but statistically significant dependence is seen.

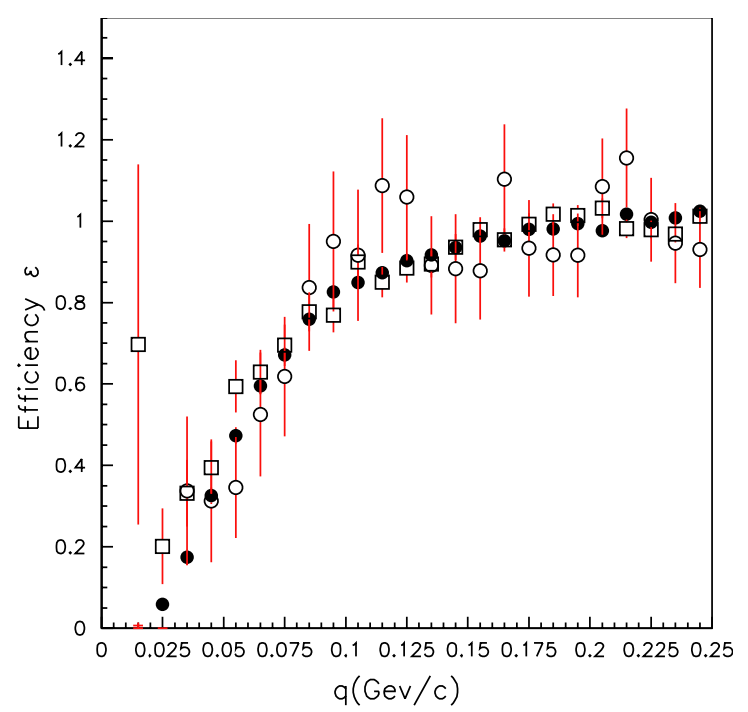

Fig. 5. The efficiency estimated by different methods for mean particle momentum $p=0.5 \mathrm{GeV} / c$. Full circles-event merging method, boxes - different mass method, open circles-Monte Carlo simulation. 


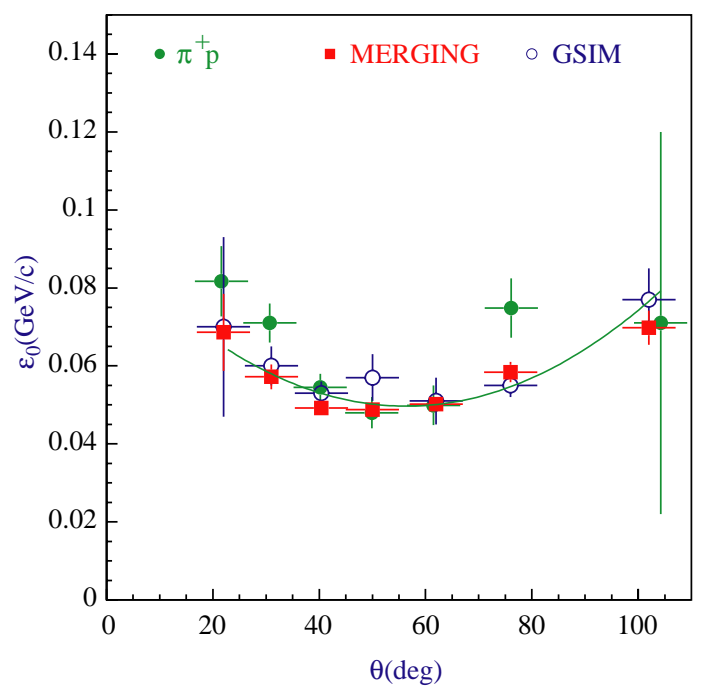

Fig. 6. The efficiency parameter $\varepsilon_{0}$ for mean particle momentum $p=0.4 \mathrm{GeV} / c$ as a function of mean emission angle $\Theta$. The curve in the figure is drawn by eye.

Fortunately, all three methods provide results compatible within statistical and systematical errors. We consider this to be a cross-check of our systematical errors.

\section{Conclusion}

(1) A new method for studying the close-track efficiency, based on the study of the correlations for particles with different masses, has been tested successfully. The advantage of the method is that it is based on the real detector hardware and software without any simulations.

(2) Another new method for studying the closetrack efficiency, which is based on the "events merging" procedure, provides results comparable with those from the other methods. The method looks very promising, because it provides the possibility to study the efficiency with excellent statistical accuracy, and because it relies on very few assumptions about the detector response.
(3) The simulation of the detector properties with the present version of GSIM is reliable with respect to the close-track reconstruction. All three methods of the close-track efficiency studying provide the same results with an accuracy sufficient for practical applications.

\section{Acknowledgements}

We particularly wish to thank Volker Burkert, Pavel Degtiarenko, Franz Klein, Georgii A. Leksin, Bernhard A. Mecking, Bogdan Niczyporuk, Nikolai Pivniouk, Stepan Stepanyan and Larry Weinstein for helpful discussions and remarks. The authors from ITEP are also pleased to acknowledge the Department of Energy, USA, for the support during our visits to the JLAB.

\section{References}

[1] G.I. Kopilov, M.I. Podgoretskii, Sov. J. Nucl. Phys. 15 (1972) 219;

S.E. Koonin, Phys. Lett. 70B (1977) 43;

R. Lednicky, V.L. Lyubo-shits, Sov. J. Nucl. Phys. 35 (1982) 770.

[2] CERN Program Library Q-123.

[3] A.V. Stavinskii, Instrum. Exp. Tech. 44-2 (2001) 164.

[4] CLAS-NOTE 2002-002, CLAS-NOTE 2003-007. (http:// www.jlab.org/Hall-B/notes).

[5] B. Mecking, et al., Nucl. Instr. and Meth. A 503 (3) (2003) 513.

[6] D.S. Carman, et al., Nucl. Instr. and Meth. A 419 (1998) 315.

[7] M.D. Mestayer, et al., Nucl. Instr. and Meth. A 449 (2000) 81.

[8] E. Smith, et al., Nucl. Instr. and Meth. A 432 (1999) 265.

[9] G. Adams, et al., Nucl. Instr. and Meth. A 465 (2001) 414.

[10] M. Amarian, et al., Nucl. Instr. and Meth. A 460 (2001) 239.

[11] M. Anghinolfi, et al., Nucl. Instr. and Meth. A 447 (2000) 424.

[12] D.H. Boal, J.C. Shillcock, Phys. Rev. C33 (1986) 549; R. Lednicky, et al., Phys. Lett. B373 (1996) 30.

[13] G.I. Kopylov, preprint JINR P2-7211(1973) (in Russian); G.I. Kopylov, Phys. Lett. B50 (1974) 472.

[14] CLAS-NOTE 1993-013 (http://www.jlab.org/Hall-B/ notes).

[15] CERN Program Library W-515. 